\title{
Integrability of Kupershmidt deformations
}

\author{
P.H.M. Kersten · I.S. Krasil'shchik · \\ A.M. Verbovetsky $\cdot$ R. Vitolo
}

\begin{abstract}
We prove that the Kupershmidt deformation of a bi-Hamiltonian system is itself bi-Hamiltonian. Moreover, Magri hierarchies of the initial system give rise to Magri hierarchies of Kupershmidt deformations as well. Since Kupershmidt deformations are not written in evolution form, we start with an outline a geometric framework to study Hamiltonian properties of general non-evolution differential equations, developed in [2] (see also [4]).
\end{abstract}

Keywords Nonlinear differential equations, variational Schouten bracket, Hamiltonian structures, symmetries, conservation laws

Mathematics Subject Classification (2000) $37 \mathrm{~K} 05 ; 35 \mathrm{Q} 53$

\section{Introduction}

Recently, in the paper [3] the authors derived the new equation

$$
\left(\partial_{x}^{3}+8 u_{x} \partial_{x}+4 u_{x x}\right)\left(u_{t}+u_{x x x}+6 u_{x}^{2}\right)=0,
$$

called the KdV6 equation, which turned out to pass the Painlevé test. The authors introduced the new variables $v=u_{x}$ and $w=u_{t}+u_{x x x}+6 u_{x}^{2}$, transforming (1) into the system

$$
v_{t}+v_{x x x}+12 v v_{x}-w_{x}=0, \quad w_{x x x}+8 v w_{x}+4 w v_{x}=0,
$$

This work was supported in part by NWO-RFBR grant 047017015 (PK, IK, and AV), RFBR-E.I.N.S.T.E.IN Consortium grant 06-01-92060 (IK, AV, and RV), and RFBR-CRNS grant 08-07-92496 (IK and AV).

P.H.M. Kersten

University of Twente, Postbus 217, 7500 AE Enschede, the Netherlands

E-mail: kerstenphm@ewi.utwente.nl

I.S. Krasil'shchik, A.M. Verbovetsky

Independent University of Moscow, B. Vlasevsky 11, 119002 Moscow, Russia

E-mail: josephk@diffiety.ac.ru, verbovet@mccme.ru

R. Vitolo

Dept. of Mathematics “E. De Giorgi”, Università del Salento, via per Arnesano, 73100 Lecce, Italy

E-mail: raffaele.vitolo@unile.it 
and posed the problem to study conservation laws and Hamiltonian structures of the above system.

In [6], Kupershmidt proved the existence of an infinite series of conservation laws for (2) in the following way. He noted that (2) can be written in the form

$$
F-A_{1}(w)=0, \quad A_{2}(w)=0,
$$

where $F=0$ is the $\mathrm{KdV}$ equation in the unknown $v$, and $A_{1}, A_{2}$ are the two standard Hamiltonian operators for $F=0$. So, (3) can be considered to be a deformation of $F=0$; we call it the Kupershmidt deformation. The following result yields an infinite series of conservation laws for (3).

Theorem (Kupershmidt) Let $F=u_{t}-f=0$ be an evolution bi-Hamiltonian system, with $A_{1}, A_{2}$ being the corresponding Hamiltonian operators. If this equation has a Magri hierarchy of conserved densities $\frac{d H_{i}}{d t}=0, A_{1}\left(\frac{\delta H_{i}}{\delta u}\right)=A_{2}\left(\frac{\delta H_{i+1}}{\delta u}\right)$, then $H_{1}, H_{2}, \ldots$ are conserved densities for (3).

Proof

$$
\begin{aligned}
\frac{d H_{i}}{d t}=\left\langle\frac{\delta H_{i}}{\delta u}, f+A_{1}(w)\right\rangle=\langle & \left.-A_{1}\left(\frac{\delta H_{i}}{\delta u}\right), w\right\rangle \\
& =\left\langle-A_{2}\left(\frac{\delta H_{i+1}}{\delta u}\right), w\right\rangle=\left\langle\frac{\delta H_{i+1}}{\delta u}, A_{2}(w)\right\rangle=0 .
\end{aligned}
$$

Further, Kupershmidt conjectured that these conservation laws commute (in a sense) so that (3) is integrable.

What makes this conjecture especially interesting is that system (3) is not in evolution form. Recently, in [2] (see also [4]) we, together with S. Igonin, have introduced a generalization of Hamiltonian formalism for general, not necessarily evolution, systems.

In the present paper, we apply this formalism to the Kupershmidt conjecture. Namely we prove that the Kupershmidt deformation of every bi-Hamiltonian equation is again a bi-Hamiltonian system and that every hierarchy of conservation laws of the original biHamiltonian system gives rise to a hierarchy of conservation laws of the Kupershmidt deformation.

\section{Preliminaries}

An adequate setting for dealing with symmetries and conservation laws of differential equations is provided by jet bundles. Our main sources are [1,5]; we will shortly describe our notation below.

From now on all manifolds and maps are $C^{\infty}$.

Jets and differential equations. Let $\pi: E \rightarrow M$ be a vector bundle. We denote by $J^{k}(\pi)$ the corresponding jet manifold and by $\pi_{k, l}: J^{k}(\pi) \rightarrow J^{l}(\pi)$ for $k>l$ and $\pi_{k}: J^{k}(\pi) \rightarrow E$ the standard projections. The inverse limit of the chain of projections $\cdots \rightarrow \pi_{k+1, k} \rightarrow \pi_{k, k-1} \rightarrow$ $\cdots$ is said to be the infinite order jet space and is denoted by $J^{\infty}(\pi)$.

Let $x^{1}, \ldots, x^{n}$ be local coordinates in $M, u^{1}, \ldots, u^{m}$ and be local fiber coordinates in $E$. Then $u_{\sigma}^{j}$, where $\sigma$ is a multiindex of arbitrary length, denote local derivative coordinates on 
the fibers of $J^{\infty}(\pi)$. If $s: M \rightarrow E$ is a section then its prolongation $j_{\infty} s: M \rightarrow J^{\infty}(\pi)$ fulfills $u_{\sigma}^{j} \circ j_{\infty} s=\partial^{|\sigma|}\left(u^{j} \circ s\right) / \partial x^{\sigma}$.

We denote by $\mathscr{F}(\pi)$ the algebra of smooth functions on $J^{\infty}(\pi)$. This is defined as the direct limit of the chain of inclusions of smooth functions on $J^{k}(\pi)$ into smooth functions on $J^{k+1}(\pi)$ via pull-back. The $\mathscr{F}(\pi)$-module $\Lambda^{*}(\pi)$ of differential forms on $J^{\infty}(\pi)$ is defined in the similar way.

A horizontal module is the $\mathscr{F}(\pi)$-module of sections of $\pi_{\infty}^{*}(\alpha)$, where $\alpha$ is a vector bundle over $M$. Denote by $\varkappa(\pi)$ the horizontal module corresponding to the bundle $\pi$ itself.

Let $P_{1}(\pi)$ and $P_{2}(\pi)$ be horizontal modules. A linear differential operator $\Delta: P_{1}(\pi) \rightarrow$ $P_{2}(\pi)$ is called $\mathscr{C}$-differential if it can be restricted to the graphs of all infinitely prolonged sections of the bundle. The set of all $\mathscr{C}$-differential operators from $P_{1}(\pi)$ to $P_{2}(\pi)$ is denoted by $\mathscr{C} \operatorname{Diff}\left(P_{1}(\pi), P_{2}(\pi)\right)$. In coordinates, $\mathscr{C}$-differential operators have the form of a matrix $\left(a_{i j}^{\sigma} D_{\sigma}\right)$, where $a_{i j}^{\sigma} \in \mathscr{F}(\pi), D_{\sigma}=D_{i_{1}} \circ \cdots \circ D_{i_{r}}$ for $\sigma=i_{1} \ldots i_{r}$ and $D_{i}=\partial / \partial x^{i}+u_{\sigma i}^{j} \partial / \partial u_{\sigma}^{j}$ is the total derivative operator with respect to $x$ 1 .

A $\pi_{\infty}$-vertical vector field on $J^{\infty}(\pi)$ is called an evolutionary field if it commutes with all $D_{i}$ (this property does not depend on the choice of coordinates). In coordinates, each evolutionary field is of the form $\vartheta_{\varphi}=D_{\sigma}\left(\varphi^{j}\right) \partial / \partial u_{\sigma}^{j}$, where $\varphi^{j} \in \mathscr{F}(\pi)$. It can be proved that each evolutionary vector field is uniquely determined by its generating function $\varphi \in$ $\varkappa(\pi)$ and vice verse to any $\varphi \in \varkappa(\pi)$ there corresponds an evolutionary field $\vartheta_{\varphi}$.

Let $P(\pi)$ be a horizontal module. For each element $p \in P(\pi)$ there is a $\mathscr{C}$-differential operator $\ell_{p}: \varkappa(\pi) \rightarrow P(\pi)$ called the universal linearization of $p$ and defined by $\ell_{p}(\varphi)=$ $\vartheta_{\varphi}(p)$, with $\varphi \in \varkappa(\pi)$. In coordinates, $\ell_{p}$ is the matrix of the form $\left(\partial p^{i} / \partial u_{\sigma}^{j} \cdot D_{\sigma}\right)$.

A differential form $\omega \in \Lambda^{q}(\pi)$ on $J^{\infty}(\pi)$ is called a Cartan form if its pull-back through any prolonged section vanishes. In coordinates, Cartan forms contain factors of the type $\omega_{\sigma}^{j}=d u_{\sigma}^{j}-u_{\sigma i}^{j} d x^{i}$. Denote the module of all Cartan $q$-forms by $\mathscr{C} \Lambda^{q}(\pi)$. It is not difficult to show that $d\left(\mathscr{C} \Lambda^{q}(\pi)\right) \subset \mathscr{C} \Lambda^{q+1}(\pi)$. Therefore the quotient $\bar{d}$ of $d$, acting on $\bar{\Lambda}^{*}(\pi)=$ $\Lambda^{*}(\pi) / \mathscr{C} \Lambda^{*}(\pi)$, is well defined. The module $\bar{\Lambda}^{*}(\pi)$ is identified with the submodule in $\Lambda^{*}(\pi)$ generated by $\pi_{\infty}^{*} \Lambda^{*}(M)$. Elements of $\bar{\Lambda}^{q}(\pi)$ are called horizontal forms. In coordinates, $\bar{\Lambda}^{q}(\pi)$ is generated by $f d x^{i_{1}} \wedge \cdots \wedge d x^{i_{q}}$, where $f \in \mathscr{F}(\pi)$, and $\bar{d}\left(f d x^{i_{1}} \wedge \cdots \wedge d x^{i_{q}}\right)=$ $D_{i}(f) d x^{i} \wedge d x^{i_{1}} \wedge \cdots \wedge d x^{i_{q}}$. The cohomology $\bar{H}^{i}(\pi)$ of the complex $\left(\bar{\Lambda}^{*}(\pi), \bar{d}\right)$ is said to be the horizontal cohomology, and coincides with the de Rham cohomology of $M$ for all degrees $i$ up to $n-1$.

If $P(\pi)$ is an $\mathscr{F}(\pi)$-module, we write $\hat{P}(\pi)=\operatorname{Hom}_{\mathscr{F}(\pi)}\left(P(\pi), \bar{\Lambda}^{n}(\pi)\right)$ and consider the natural pairing $\langle\cdot, \cdot\rangle: \hat{P}(\pi) \times P(\pi) \rightarrow \bar{\Lambda}^{n}(\pi)$. We recall that for each operator $\Delta \in$ $\mathscr{C} \operatorname{Diff}\left(P_{1}(\pi), P_{2}(\pi)\right)$ there exists a unique operator $\Delta^{*} \in \mathscr{C} \operatorname{Diff}\left(\hat{P}_{2}(\pi), \hat{P}_{1}(\pi)\right)$ such that

$$
\left[\left\langle\hat{p}_{2}, \Delta\left(p_{1}\right)\right\rangle\right]=\left[\left\langle\Delta^{*}\left(\hat{p}_{2}\right), p_{1}\right\rangle\right], \quad \hat{p}_{2} \in \hat{P}_{2}(\pi), \quad p_{1} \in P_{1}(\pi),
$$

where $[\omega]$ denotes the horizontal cohomology class of $\omega \in \bar{\Lambda}^{n}$. The operator $\Delta^{*}$ is called adjoint to $\Delta$. In coordinates, $\left(a_{i j}^{\sigma} D_{\sigma}\right)^{*}=\left((-1)^{|\sigma|} D_{\sigma} \circ a_{j i}^{\sigma}\right)$, where $a_{i j}^{\sigma} \in \mathscr{F}(\pi)$.

A differential equation is a submanifold $\mathscr{E} \subset J^{k}(\pi)$. We assume that $\mathscr{E}=\{F=0\}$, where $F \in \pi_{k}^{*}(\alpha)$ is a section of the pull-back bundle of a vector bundle $\alpha$ on $M$.

Let us set $P(\pi)=\pi_{\infty}^{*}(\alpha)$. The equation $\mathscr{E}$ can be prolonged to a submanifold of $J^{\infty}(\pi)$, that we still denote by $\mathscr{E}$. In local coordinates, we have $\mathscr{E}=\left\{D_{\sigma} F^{k}=0\right\}$ for such a prolongation. Throughout the paper we will assume equations to be regular: this means that any function $f \in \mathscr{F}(\pi)$ vanishing on $\mathscr{E}$ can be expressed as $f=\Delta(F)$, where $\Delta \in \mathscr{C} \operatorname{Diff}(P(\pi), \mathscr{F}(\pi))$.

1 The Einstein summation convention will be used throughout the paper. 
Symmetries. For any horizontal module $Q(\pi)$ we will denote by $Q$ the restriction of $Q(\pi)$ to $\mathscr{E}$. We denote by $\ell_{\mathscr{E}}: \varkappa \rightarrow P$ the restriction of the universal linearization $\ell_{F}$ to $\mathscr{E}$. The space $\operatorname{ker} \ell_{\mathscr{E}}$ coincides with the space $\operatorname{Sym} \mathscr{E}$ of symmetries of the differential equation $\mathscr{E}$. The space $\operatorname{ker} \ell_{\mathscr{E}}^{*}$ is the space of cosymmetries, and is denoted by $\operatorname{CoSym} \mathscr{E}$.

It can be proved that there exists a sequence

$$
0 \rightarrow \mathscr{C} \operatorname{Diff}(P, \mathscr{F}) \rightarrow \mathscr{C} \operatorname{Diff}(\varkappa, \mathscr{F}) \rightarrow \mathscr{C} \Lambda^{1} \rightarrow 0
$$

Here the first nontrivial map is defined by $\Delta \mapsto \Delta \circ \ell_{\mathscr{E}}$ and the second one is a natural projection (see [1,5] for details). The differential equation $\mathscr{E}$ is said to be normal if the above sequence is exact. In other words, $\mathscr{E}$ is normal, if the equality $\Delta \circ \ell_{\mathscr{E}}$ implies $\Delta=0$. Most differential equations of mathematical physics fulfill this property; however, the gauge equations do not.

Conservation laws. Consider the horizontal de Rham complex $\left(\bar{\Lambda}^{q}, \bar{d}\right)$ on $\mathscr{E}$. A conservation law is an element $[\omega] \in \bar{H}^{n-1} / H^{n-1}(M)$ (we quotient out the topological conservation laws). The Vinogradov $\mathscr{C}$-spectral sequence, see [1,5,7] for a detailed treatment) yields the complex $\left(E_{1}^{*, n-1}, d_{1}\right)$ which plays the same role as the de Rham complex on jets. In particular the first two terms of the complex are $E_{1}^{0, n-1}=\bar{H}^{n-1}$ and $E_{1}^{1, n-1}=\operatorname{ker} \ell_{\mathscr{E}}^{*}=\operatorname{CoSym} \mathscr{E}$, and the first differential $d_{1}: E_{1}^{0, n-1} \rightarrow E_{1}^{1, n-1}$ is defined by $d_{1}([\omega])=\Delta^{*}(1)$, where $\Delta \in$ $\mathscr{C} \operatorname{Diff}\left(P, \bar{\Lambda}^{n}\right)$ fulfills $\bar{d} \omega=\Delta(F)$. The element $\Delta^{*}(1) \in \hat{P}$ is said to be a generating function of the conservation law $[\omega]$.

A $\mathscr{C}$-spectral sequence argument shows that there exists an exact sequence $0 \rightarrow H^{n-1} \rightarrow$ $\bar{H}^{n-1} \rightarrow \operatorname{ker} \ell_{\mathscr{E}}^{*}$, where the last map is just $d_{1}$. So, the space of conservation laws $\operatorname{cl}(\mathscr{E})=$ $\bar{H}^{n-1} / H^{n-1}(M)$ is a subset of the kernel of $\ell_{\mathscr{E}}^{*}, \operatorname{cl}(\mathscr{E}) \subset \operatorname{ker} \ell_{\mathscr{E}}^{*}=\operatorname{CoSym} \mathscr{E}$.

\section{Hamiltonian bivectors on general equations}

In this section we collect formulas related to the Hamiltonian formalism on general equations. For details and geometric definitions we refer the reader to [2] (see also [4]).

Let $\mathscr{E} \subset J^{\infty}(\pi)$ be a normal equation given by $F=0$.

A variational bivector on $\mathscr{E}$ is the equivalence class of $\mathscr{C}$-differential operators $A: \hat{P} \rightarrow$ $\varkappa$ on $\mathscr{E}$ that satisfy the condition

$$
\ell_{\mathscr{E}} \circ A=A^{*} \circ \ell_{\mathscr{E}}^{*}
$$

where two operators are equivalent if they differ by an operator of the form $\square \circ \ell_{\mathscr{E}}^{*}, \square=$ $\square^{*}: \hat{\varkappa} \rightarrow \varkappa$.

It is straightforward to see that an action of variational bivectors on cosymmetries (and, in particular, on conservation laws) is well-defined and the result is a symmetry.

If $A$ is a bivector then on $J^{\infty}(\pi)$ we have

$$
\ell_{F} \circ A-A^{*} \circ \ell_{F}^{*}=B(F, \cdot),
$$

where $A$ is extended onto $J^{\infty}(\pi), B: P(\pi) \times \hat{P}(\pi) \rightarrow P(\pi)$ is a $\mathscr{C}$-differential operator. We denote by $B^{*}: \hat{P} \times \hat{P} \rightarrow \hat{P}$ the operator adjoint to the operator $B$ in the first argument and restricted to $\mathscr{E}$. 
Consider an equivalence relation on the set of operators $\mathscr{C} \operatorname{Diff}(\hat{P} \times \hat{P}, \hat{P})$ on $\mathscr{E}$ such that two operators are equivalent if they differ by an operator of the form

$$
\square_{1}\left(\ell_{\mathscr{E}}^{*}(\cdot), \cdot\right)+\square_{2}\left(\cdot, \ell_{\mathscr{E}}^{*}(\cdot)\right),
$$

where $\square_{1}: \hat{\varkappa} \times \hat{P} \rightarrow \hat{P}, \square_{2}: \hat{P} \times \hat{\varkappa} \rightarrow \hat{P}$

Proposition 1 For every bivector $A$ the equivalence class of $B^{*}$ is uniquely defined and contains a skew-symmetric operator $B^{*}: \hat{P} \times \hat{P} \rightarrow \hat{P}$.

Remark 1 If $\mathscr{E}$ is written in evolution form (in this case $P=\varkappa$ ) then we can put $B^{*}\left(\psi_{1}, \psi_{2}\right)=$ $\ell_{A, \psi_{2}}^{*}\left(\psi_{1}\right)$, here we use the notation $\ell_{\Delta, p}=\ell_{\Delta(p)}-\Delta \circ \ell_{p}$. Skew-symmetricity follows from the formula

$$
\ell_{\Delta, p}^{*}(\hat{q})=\ell_{\Delta^{*}, \hat{q}}^{*}(p) .
$$

The Schouten bracket of two bivectors is defined by the formula

$$
\begin{aligned}
& \|\left[A_{1}, A_{2}\right]\left(\psi_{1}, \psi_{2}\right) \\
& \quad=\ell_{A_{1}, \psi_{1}}\left(A_{2}\left(\psi_{2}\right)\right)-\ell_{A_{1}, \psi_{2}}\left(A_{2}\left(\psi_{1}\right)\right) \\
& \quad+\ell_{A_{2}, \psi_{1}}\left(A_{1}\left(\psi_{2}\right)\right)-\ell_{A_{2}, \psi_{2}}\left(A_{1}\left(\psi_{1}\right)\right) \\
& \quad \quad-A_{1}\left(B_{2}^{*}\left(\psi_{1}, \psi_{2}\right)\right)-A_{2}\left(B_{1}^{*}\left(\psi_{1}, \psi_{2}\right)\right),
\end{aligned}
$$

where $\psi_{1}, \psi_{2} \in \hat{P}$ and $B_{1}, B_{2}$ are the operators which play the role of $B$ in Eq. (6) for $A_{1}, A_{2}$, respectively.

The Schouten bracket is a bracket between multivectors. This means that it is defined on a wide class of operators. In particular, 0 -vectors are conservation laws and the bracket between a variational bivector $A$ and a conservation law $\omega$ has the form

$$
[[A, \omega]]=A(\psi),
$$

where $\psi=d_{1}[\omega]$ is the generating function of $\omega ; 1$-vectors are symmetries, the bracket between a symmetry $\varphi$ and a conservation law $[\omega]$ :

$$
\llbracket \varphi, \omega]=L_{\varphi}(\omega)=\vartheta_{\varphi}(\psi)+\Delta^{*}(\psi),
$$

where $L_{\varphi}$ is the Lie derivative and the operator $\Delta: P \rightarrow P$ is defined by the relation $\ell_{F}(\varphi)=$ $\Delta(F)$ on $J^{\infty}$ (for equations in evolution form one can take $\Delta=\ell_{\varphi}$ ); the bracket between two symmetries

$$
\left.\llbracket \varphi_{1}, \varphi_{2}\right]=\left[\varphi_{1}, \varphi_{2}\right] \quad \text { (the usual commutator) }
$$

and the bracket between two conservation laws

$$
\llbracket \omega_{1}, \omega_{2} \rrbracket=0 .
$$

A bivector $A$ is called Hamiltonian if $[A, A]]=0$.

A Hamiltonian bivector $A$ on $\mathscr{E}$ gives rise to a Lie algebra structure on the space of conservation laws of $\mathscr{E}:$

$$
\left\{\omega_{1}, \omega_{2}\right\}_{A}=\llbracket\left[\left[A, \omega_{1}\right]\right], \omega_{2} \rrbracket .
$$

An equation $\mathscr{E}$ is called bi-Hamiltonian if it has two Hamiltonian bivectors $A_{1}$ and $A_{2}$ such that $\left[\left[A_{1}, A_{2}\right]\right]=0$.

Magri hierarchy on a bi-Hamiltonian equation $\mathscr{E}$ is an infinite sequence $\omega_{1}, \omega_{2}, \ldots$ of conservation laws of $\mathscr{E}$ such that $A_{1}\left(\psi_{i}\right)=A_{2}\left(\psi_{i+1}\right)$. 
Proposition 2 For Magri hierarchy we get

$$
\begin{gathered}
\left\{\omega_{i}, \omega_{j}\right\}_{A_{1}}=0, \quad\left\{\omega_{i}, \omega_{j}\right\}_{A_{2}}=0 \\
\left\{\varphi_{i}, \varphi_{j}\right\}=0
\end{gathered}
$$

where $\varphi_{i}=A_{1}\left(\psi_{i}\right)=A_{2}\left(\psi_{i+1}\right)$ are symmetries and the bracket between them is the commutator: $\vartheta_{\left\{\varphi_{i}, \varphi_{j}\right\}}=\left[\vartheta_{\varphi_{i}}, \vartheta_{\varphi_{2}}\right]$.

Remark 2 The reader is invited to check that, if $\mathscr{E}$ has an evolutionary form, then the above Hamiltonian formalism reduces to the usual one.

\section{The Kupershmidt deformation}

Let $\mathscr{E}$ be a bi-Hamiltonian equation with Hamiltonian operators $A_{1}$ and $A_{2}$ given by $F=0$ as above. Let us consider the bundle $\hat{\pi}: \hat{E}=E^{*} \otimes_{M} \Lambda^{n}\left(T^{*} M\right) \rightarrow M$, where $E^{*} \rightarrow M$ is the dual bundle to $\pi: E \rightarrow M$. We denote by $w=\left(w^{1}, \ldots, w^{m}\right)$ fiber coordinates on $\hat{\pi}$.

Definition 1 The Kupershmidt deformation $\tilde{\mathscr{E}} \subset J^{\infty}(\pi) \times J^{\infty}(\hat{\pi})$ has the form

$$
F+A_{1}^{*}(w)=0, \quad A_{2}^{*}(w)=0 .
$$

We will write $\tilde{F}=\left(F+A_{1}^{*}(w), A_{2}^{*}(w)\right) \in \tilde{P}=P \oplus P$, so that $\tilde{\mathscr{E}}$ is given by $\tilde{F}=0$.

The linearization $\ell_{\tilde{\mathscr{E}}}: \varkappa \oplus \hat{\varkappa} \rightarrow P \oplus P$ and its adjoint have the form

$$
\ell_{\tilde{\mathscr{E}}}=\left(\begin{array}{cc}
\ell_{F+A_{1}^{*}(w)} & A_{1}^{*} \\
\ell_{A_{2}^{*}(w)} & A_{2}^{*}
\end{array}\right), \quad \ell_{\tilde{\mathscr{E}}}^{*}=\left(\begin{array}{cc}
\ell_{F+A_{1}^{*}(w)}^{*} & \ell_{A_{2}^{*}(w)}^{*} \\
A_{1} & A_{2}
\end{array}\right)
$$

The linearizations in the left- and right-hand sides of these formulas have different meaning: the left-hand ones are usual linearizations on $\tilde{\mathscr{E}}$, while the right-hand ones are linearizations with respect to the dependent variables $u$ only, that is, linearizations on $J^{\infty}(\pi)$.

In what follow we use the following notational rule: linearization of something marked with tilde is the $J^{\infty}(\pi) \times J^{\infty}(\hat{\pi})$-linearization (i.e., the linearization with respect to the dependent variables $u, w)$, otherwise it is the $J^{\infty}(\pi)$-linearization.

Below we often use the obvious relation $\ell_{A^{*}, w}=\ell_{A^{*}(w)}$, which is true since the linearization here is the $J^{\infty}(\pi)$-linearization.

Lemma 1 We have the following equalities:

$$
\begin{aligned}
& \ell_{F+A_{1}^{*}(w)} \circ A_{1}-A_{1}^{*} \circ \ell_{F+A_{1}^{*}(w)}^{*}=B_{1}\left(F+A_{1}^{*}(w), \cdot\right) \\
& \begin{aligned}
\ell_{F+A_{1}^{*}(w)} \circ A_{2}-A_{1}^{*} \circ \ell_{A_{2}^{*}(w)}^{*}-A_{2}^{*} \circ \ell_{F+A_{1}^{*}(w)}^{*} & -\ell_{A_{2}^{*}(w)} \circ A_{1} \\
& =B_{2}\left(F+A_{1}^{*}(w), \cdot\right)+B_{1}\left(A_{2}^{*}(w), \cdot\right)
\end{aligned} \\
& \ell_{A_{2}^{*}(w)} \circ A_{2}-A_{2}^{*} \circ \ell_{A_{2}^{*}(w)}^{*}=B_{2}\left(A_{2}^{*}(w), \cdot\right) .
\end{aligned}
$$

Proof We will only prove the statement for the first operator. For every $\psi_{1}, \psi_{2} \in \hat{P}$ we have the following equalities modulo im $\bar{d}$ :

$$
\begin{aligned}
& \left\langle\ell_{F}\left(A_{1}\left(\psi_{1}\right)\right)+\ell_{A_{1}^{*}, w}\left(A_{1}\left(\psi_{1}\right)\right), \psi_{2}\right\rangle-\left\langle\ell_{F}\left(A_{1}\left(\psi_{2}\right)\right)+\ell_{A_{1}^{*}, w}\left(A_{1}\left(\psi_{2}\right)\right), \psi_{1}\right\rangle \\
& =\left\langle\left(\ell_{F} \circ A_{1}-A_{1}^{*} \circ \ell_{F}^{*}\right)\left(\psi_{1}\right), \psi_{2}\right\rangle+\left\langle A_{1}\left(\psi_{1}\right), \ell_{A_{1}^{*}, w}^{*}\left(\psi_{2}\right)\right\rangle-\left\langle A_{1}\left(\psi_{2}\right), \ell_{A_{1}^{*}, w}^{*}\left(\psi_{1}\right)\right\rangle
\end{aligned}
$$




$$
\begin{aligned}
& =\left\langle B_{1}\left(F, \psi_{1}\right), \psi_{2}\right\rangle+\left\langle A_{1}\left(\psi_{1}\right), \ell_{A_{1}, \psi_{2}}^{*}(w)\right\rangle-\left\langle A_{1}\left(\psi_{2}\right), \ell_{A_{1}, \psi_{1}}^{*}(w)\right\rangle \\
& =\left\langle B_{1}^{*}\left(\psi_{2}, \psi_{1}\right), F\right\rangle+\left\langle\left(\ell_{A_{1}, \psi_{2}}\left(A_{1}\left(\psi_{1}\right)\right)-\ell_{A_{1}, \psi_{1}}\left(A_{1}\left(\psi_{2}\right)\right)\right), w\right\rangle \\
& =\left\langle B_{1}^{*}\left(\psi_{2}, \psi_{1}\right), F\right\rangle+\left\langle A_{1}\left(B_{1}^{*}\left(\psi_{2}, \psi_{1}\right)\right), w\right\rangle \\
& =\left\langle B_{1}^{*}\left(\psi_{2}, \psi_{1}\right), F+A_{1}^{*}(w)\right\rangle \\
& =\left\langle B_{1}\left(F+A_{1}^{*}(w), \psi_{1}\right), \psi_{2}\right\rangle .
\end{aligned}
$$

The equalities in the statement turn out to be true in view of the fact that both sides of the above equalities are linear (zero order) operators in $\psi_{2}$, and the only linear operator which is the composition of $\bar{d}$ and an operator in $\psi_{2}$ is the zero operator.

The other two equalities are proved similarly.

Of course the right-hand sides of the equations in Lemma 1 vanish on $\tilde{\mathscr{E}}$, hence the following corollary.

Corollary 1 The following operators are selfadjoint on $\tilde{\mathscr{E}}$ :

$$
\begin{aligned}
& \ell_{F} \circ A_{1}+\ell_{A_{1}^{*}(w)} \circ A_{1} \\
& \ell_{F} \circ A_{2}+\ell_{A_{1}^{*}(w)} \circ A_{2}-A_{1}^{*} \circ \ell_{A_{2}^{*}, w}^{*} \\
& \ell_{A_{2}^{*}(w)} \circ A_{2} .
\end{aligned}
$$

In our computations we will also need the linearization of Eqs. (12).

Lemma 2 The linearization of Eqs. (12) computed in $\psi_{1} \in \hat{P}$ yields the equations

$$
\begin{aligned}
& \ell_{\ell_{F+A_{1}^{*}(w)}, A_{1}\left(\psi_{1}\right)}+\ell_{F+A_{1}^{*}(w)} \circ \ell_{A_{1}, \psi_{1}}-\ell_{A_{1}^{*}, \ell_{F+A_{1}^{*}(w)}^{*}}\left(\psi_{1}\right)-A_{1}^{*} \circ \ell_{\ell_{F+A_{1}^{*}(w)}^{*}, \psi_{1}} \\
& =B_{1}\left(\cdot, \psi_{1}\right) \circ \ell_{F+A_{1}^{*}(w)} \\
& \ell_{\ell_{F+A_{1}^{*}(w)}, A_{2}\left(\psi_{1}\right)}+\ell_{F+A_{1}^{*}(w)} \circ \ell_{A_{2}, \psi_{1}}-\ell_{A_{1}^{*}, \ell_{A_{2}^{*}(w)}^{*}\left(\psi_{1}\right)}-A_{1}^{*} \circ \ell_{{A_{2}^{*}(w)}_{*}^{*}, \psi_{1}} \\
& -\ell_{A_{2}^{*}, \ell_{F+A_{1}^{*}(w)}^{*}\left(\psi_{1}\right)}-A_{2}^{*} \circ \ell_{\ell_{F+A_{1}^{*}(w)}^{*}, \psi_{1}}+\ell_{\ell_{A_{2}^{*}(w)}, A_{1}\left(\psi_{1}\right)}+\ell_{A_{2}^{*}(w)} \circ \ell_{A_{1}, \psi_{1}} \\
& =B_{2}\left(\cdot, \psi_{1}\right) \circ \ell_{F+A_{1}^{*}(w)}+B_{1}\left(\cdot, \psi_{1}\right) \circ \ell_{A_{2}^{*}(w)} \\
& \ell_{\ell_{A_{2}^{*}(w)}, A_{2}\left(\psi_{1}\right)}+\ell_{A_{2}^{*}(w)} \circ \ell_{A_{2}, \psi_{1}}-\ell_{A_{2}^{*}, \ell_{A_{2}^{*}(w)}^{*}\left(\psi_{1}\right)}-A_{2}^{*} \circ \ell_{\ell_{2}^{*}(w)}, \psi_{1}=B_{2}\left(\cdot, \psi_{1}\right) \circ \ell_{A_{2}^{*}(w)}
\end{aligned}
$$

up to terms which vanish on $\tilde{\mathscr{E}}$.

Proof We make use of the formula

$$
\ell_{\Delta \circ \square(\alpha)}=\ell_{\Delta, \square(\alpha)}+\Delta \circ \ell_{\square, \alpha}+\Delta \circ \square \circ \ell_{\alpha} .
$$

Let us apply the above formula to Eq. $12 \mathrm{a}$ computed at $\psi_{1}$ :

$$
\begin{aligned}
\ell_{\ell_{F+A_{1}^{*}(w)}, A_{1}\left(\psi_{1}\right)}+ & \ell_{F+A_{1}^{*}(w)} \circ \ell_{A_{1}, \psi_{1}}+\ell_{F+A_{1}^{*}(w)} \circ A_{1} \circ \ell_{\psi_{1}} \\
& -\ell_{A_{1}^{*}, \ell_{F+A_{1}^{*}(w)}^{*}\left(\psi_{1}\right)}-A_{1}^{*} \circ \ell_{\ell_{F+A_{1}^{*}(w)}^{*}, \psi_{1}}-A_{1}^{*} \circ \ell_{F+A_{1}^{*}(w)}^{*} \circ \ell_{\psi_{1}} \\
& =\ell_{B_{1}(\cdot, \cdot),\left(F+A_{1}^{*}(w), \psi_{1}\right)}+B_{1}\left(\cdot, \psi_{1}\right) \circ \ell_{F+A_{1}^{*}(w)}+B_{1}\left(F+A_{1}^{*}(w), \cdot\right) \circ \ell_{\psi_{1}} .
\end{aligned}
$$

We obtain Eq. 13a by observing that the term $\ell_{B_{1}(\cdot, \cdot),\left(F+A_{1}^{*}(w), \psi_{1}\right)}$ vanishes on $\tilde{E}$ and that terms composed with $\ell_{\psi_{1}}$ cancel by virtue of Eq. (12a). The two remaining equations can be derived with similar reasoning. 
Now let us consider the operators $\tilde{A}_{1}, \tilde{A}_{2}: \hat{P} \oplus \hat{P} \rightarrow \varkappa \oplus \hat{\varkappa}$ defined by

$$
\tilde{A}_{1}=\left(\begin{array}{cc}
A_{1} & -A_{1} \\
0 & \ell_{F+A_{1}^{*}(w)+A_{2}^{*}(w)}^{*}
\end{array}\right), \quad \tilde{A}_{2}=\left(\begin{array}{cc}
A_{2} & -A_{2} \\
-\ell_{F+A_{1}^{*}(w)+A_{2}^{*}(w)} & 0
\end{array}\right) .
$$

Proposition 3 We have the equalities

$$
\begin{aligned}
& \ell_{\tilde{F}} \circ \tilde{A}_{1}-\tilde{A}_{1}^{*} \circ \ell_{\tilde{F}}^{*}=\tilde{B}_{1}(\tilde{F}, \cdot) \\
& \ell_{\tilde{F}} \circ \tilde{A}_{2}-\tilde{A}_{2}^{*} \circ \ell_{\tilde{F}}^{*}=\tilde{B}_{2}(\tilde{F}, \cdot)
\end{aligned}
$$

where

$$
\begin{aligned}
& \tilde{B}_{1}\left(\left(p_{1}, p_{1}^{\prime}\right),\left(\psi_{2}, \psi_{2}^{\prime}\right)\right)= \\
& \quad\left(B_{1}\left(p_{1}, \psi_{2}\right)-B_{1}\left(p_{1}, \psi_{2}^{\prime}\right),-B_{1}\left(p_{1}, \psi_{2}\right)-B_{2}\left(p_{1}, \psi_{2}^{\prime}\right)-B_{1}\left(p_{1}^{\prime}, \psi_{2}^{\prime}\right)-B_{2}\left(p_{1}^{\prime}, \psi_{2}^{\prime}\right)\right) \\
& \tilde{B}_{2}\left(\left(p_{1}, p_{1}^{\prime}\right),\left(\psi_{2}, \psi_{2}^{\prime}\right)\right)= \\
& \quad\left(B_{1}\left(p_{1}, \psi_{2}\right)+B_{2}\left(p_{1}, \psi_{2}\right)+B_{1}\left(p_{1}^{\prime}, \psi_{2}\right)+B_{2}\left(p_{1}^{\prime}, \psi_{2}^{\prime}\right), B_{2}\left(p_{1}^{\prime}, \psi_{2}\right)-B_{2}\left(p_{1}^{\prime}, \psi_{2}^{\prime}\right)\right)
\end{aligned}
$$

It follows that the operators $\tilde{A}_{1}, \tilde{A}_{2}$ define two variational bivectors on $\tilde{E}$.

Proof We have

$$
\begin{aligned}
& \ell_{\tilde{F}} \circ \tilde{A}_{1}-\tilde{A}_{1}^{*} \circ \ell_{\tilde{F}}^{*} \\
& =\left(\begin{array}{cc}
\ell_{F+A_{1}^{*}(w)} & A_{1}^{*} \\
\ell_{A_{2}^{*}(w)} & A_{2}^{*}
\end{array}\right) \cdot\left(\begin{array}{cc}
A_{1} & -A_{1} \\
0 & \ell_{F+A_{1}^{*}(w)+A_{2}^{*}(w)}^{*}
\end{array}\right) \\
& -\left(\begin{array}{cc}
A_{1}^{*} & 0 \\
-A_{1}^{*} & \ell_{F+A_{1}^{*}(w)+A_{2}^{*}(w)}
\end{array}\right) \cdot\left(\begin{array}{cc}
\ell_{F+A_{1}^{*}(w)}^{*} & \ell_{A_{2}^{*}(w)}^{*} \\
A_{1} & A_{2}
\end{array}\right) \\
& =\left(\begin{array}{cc}
B_{1}\left(F+A_{1}^{*}(w), \cdot\right) & -B_{1}\left(F+A_{1}^{*}(w), \cdot\right) \\
-B_{1}\left(F+A_{1}^{*}(w), \cdot\right) & -B_{2}\left(F+A_{1}^{*}(w), \cdot\right)-\left(B_{1}+B_{2}\right)\left(A_{2}^{*}(w), \cdot\right)
\end{array}\right),
\end{aligned}
$$

where the last equality is obtained by Lemma 1 Eqs. (16) and (18) can be proved in the same way.

The last statement follows directly from the definitions (Eqs. (5) and (6)).

Our next task is to prove that the bivectors from the above proposition endow $\tilde{\mathscr{E}}$ with a bi-Hamiltonian structure. To this aim we have to compute the Schouten brackets $\llbracket \tilde{A}_{i}, \tilde{A}_{j} \rrbracket$ for $i, j=1,2$. To do this, we have to compute the linearization $\ell_{\tilde{A}_{i},\left(\psi_{1}, \psi_{1}^{\prime}\right)}$ and the operators $\tilde{B}_{i}^{*}$, with $i=1,2$ (here the adjoint is taken with respect to the first argument). We have

$$
\ell_{\tilde{A}_{1},\left(\psi_{1}, \psi_{1}^{\prime}\right)}=\left(\begin{array}{cc}
\ell_{A_{1}, \psi_{1}}-\ell_{A_{1}, \psi_{1}^{\prime}} & 0 \\
\ell_{\ell_{F+A_{1}^{*}(w)+A_{2}^{*}(w)}, \psi_{1}^{\prime}} & \ell_{A_{1}+A_{2}, \psi_{1}^{\prime}}^{*}
\end{array}\right),
$$

and

$$
\ell_{\tilde{A}_{2},\left(\psi_{1}, \psi_{1}^{\prime}\right)}=\left(\begin{array}{cc}
\ell_{A_{2}, \psi_{1}}-\ell_{A_{2}, \psi_{1}^{\prime}} & 0 \\
-\ell_{\ell_{F+A_{1}^{*}(w)+A_{2}^{*}(w)}^{*}, \psi_{1}} & -\ell_{A_{1}+A_{2}, \psi_{1}}^{*}
\end{array}\right)
$$

directly from the definitions. 
Lemma 3 We have

$$
\begin{aligned}
& \tilde{B}_{1}^{*}\left(\left(\psi_{1}, \psi_{1}^{\prime}\right),\left(\psi_{2}, \psi_{2}^{\prime}\right)\right) \\
& \quad=\left(B_{1}^{*}\left(\psi_{1}, \psi_{2}\right)-B_{1}^{*}\left(\psi_{1}, \psi_{2}^{\prime}\right)-B_{1}^{*}\left(\psi_{1}^{\prime}, \psi_{2}\right)-B_{2}^{*}\left(\psi_{1}^{\prime}, \psi_{2}^{\prime}\right),-B_{1}^{*}\left(\psi_{1}^{\prime}, \psi_{2}^{\prime}\right)-B_{2}^{*}\left(\psi_{1}^{\prime}, \psi_{2}^{\prime}\right)\right)
\end{aligned}
$$

$$
\begin{aligned}
& \tilde{B}_{2}^{*}\left(\left(\psi_{1}, \psi_{1}^{\prime}\right),\left(\psi_{2}, \psi_{2}^{\prime}\right)\right) \\
& \quad=\left(B_{1}^{*}\left(\psi_{1}, \psi_{2}\right)+B_{2}^{*}\left(\psi_{1}, \psi_{2}\right), B_{1}^{*}\left(\psi_{1}, \psi_{2}\right)+B_{2}^{*}\left(\psi_{1}, \psi_{2}^{\prime}\right)+B_{2}^{*}\left(\psi_{1}^{\prime}, \psi_{2}\right)-B_{2}^{*}\left(\psi_{1}^{\prime}, \psi_{2}^{\prime}\right)\right)
\end{aligned}
$$

Proof In fact we can write

$$
\tilde{B}_{1}\left(\left(p_{1}, p_{1}^{\prime}\right),\left(\psi_{2}, \psi_{2}^{\prime}\right)\right)=\left(\begin{array}{cc}
B_{1}\left(\cdot, \psi_{2}\right)-B_{1}\left(\cdot, \psi_{2}^{\prime}\right) & 0 \\
-B_{1}\left(\cdot, \psi_{2}\right)-B_{2}\left(\cdot, \psi_{2}^{\prime}\right) & -B_{1}\left(\cdot, \psi_{2}^{\prime}\right)-B_{2}\left(\cdot, \psi_{2}^{\prime}\right)
\end{array}\right) \cdot\left(\begin{array}{l}
p_{1} \\
p_{1}^{\prime}
\end{array}\right) .
$$

It follows that

$$
\tilde{B}_{1}^{*}\left(\left(\psi_{1}, \psi_{1}^{\prime}\right),\left(\psi_{2}, \psi_{2}^{\prime}\right)\right)=\left(\begin{array}{cc}
B_{1}^{*}\left(\cdot, \psi_{2}\right)-B_{1}^{*}\left(\cdot, \psi_{2}^{\prime}\right) & -B_{1}^{*}\left(\cdot, \psi_{2}\right)-B_{2}^{*}\left(\cdot, \psi_{2}^{\prime}\right) \\
0 & -B_{1}^{*}\left(\cdot, \psi_{2}^{\prime}\right)-B_{2}^{*}\left(\cdot, \psi_{2}^{\prime}\right)
\end{array}\right) \cdot\left(\begin{array}{l}
\psi_{1} \\
\psi_{1}^{\prime}
\end{array}\right) .
$$

The other identity follows in an analogous way.

Note that $\tilde{B}_{1}^{*}$ and $\tilde{B}_{2}^{*}$ are skew-symmetric with respect to the interchange of the arguments $\left(\psi_{1}, \psi_{1}^{\prime}\right)$ and $\left(\psi_{2}, \psi_{2}^{\prime}\right)$.

Theorem 1 The Kupershmidt deformation $\tilde{\mathscr{E}}$ is a bi-Hamiltonian equation with respect to the variational bivectors $\tilde{A}_{1}, \tilde{A}_{2}$.

Proof We have to prove the conditions

$$
\begin{aligned}
& {\left[\left[\tilde{A}_{i}, \tilde{A}_{j}\right]\right]\left(\left(\psi_{1}, \psi_{1}^{\prime}\right),\left(\psi_{2}, \psi_{2}^{\prime}\right)\right)} \\
& \quad=\ell_{\tilde{A}_{i},\left(\psi_{1}, \psi_{1}^{\prime}\right)}\left(\tilde{A}_{j}\left(\psi_{2}, \psi_{2}^{\prime}\right)\right)-\ell_{\tilde{A}_{i},\left(\psi_{2}, \psi_{2}^{\prime}\right)}\left(\tilde{A}_{j}\left(\psi_{1}, \psi_{1}^{\prime}\right)\right) \\
& \quad+\ell_{\tilde{A}_{j},\left(\psi_{1}, \psi_{1}^{\prime}\right)}\left(\tilde{A}_{i}\left(\psi_{2}, \psi_{2}^{\prime}\right)\right)-\ell_{\tilde{A}_{j},\left(\psi_{2}, \psi_{2}^{\prime}\right)}\left(\tilde{A}_{i}\left(\psi_{1}, \psi_{1}^{\prime}\right)\right) \\
& \quad-\tilde{A}_{i}\left(\tilde{B}_{j}^{*}\left(\left(\psi_{1}, \psi_{1}^{\prime}\right),\left(\psi_{2}, \psi_{2}^{\prime}\right)\right)\right)-\tilde{A}_{j}\left(\tilde{B}_{i}^{*}\left(\left(\psi_{1}, \psi_{1}^{\prime}\right),\left(\psi_{2}, \psi_{2}^{\prime}\right)\right)\right)=0,
\end{aligned}
$$

for $i, j=1,2$. We will only prove them for $i=1, j=2$, other computations being very similar. First of all we compute the summands in the expression 23):

$$
\begin{aligned}
& \ell_{\tilde{A}_{1},\left(\psi_{1}, \psi_{1}^{\prime}\right)}\left(\tilde{A}_{2}\left(\psi_{2}, \psi_{2}^{\prime}\right)\right) \\
& =\left(\left(\ell_{A_{1}, \psi_{1}}-\ell_{A_{1}, \psi_{1}^{\prime}}\right)\left(A_{2}\left(\psi_{2}\right)-A_{2}\left(\psi_{2}^{\prime}\right)\right)\right. \text {, } \\
& \left.\mathscr{L}\left(\psi_{1}, A_{2}\left(\psi_{2}\right)-A_{2}\left(\psi_{2}^{\prime}\right)\right)+\ell_{A_{1}+A_{2}, \psi_{1}}^{*}\left(-\ell_{F+A_{1}^{*}(w)+A_{2}^{*}(w)}^{*}\left(\psi_{2}^{\prime}\right)\right)\right), \\
& \ell_{\tilde{A}_{2},\left(\psi_{1}, \psi_{1}^{\prime}\right)}\left(\tilde{A}_{1}\left(\psi_{2}, \psi_{2}^{\prime}\right)\right) \\
& =\left(\left(\ell_{A_{2}, \psi_{1}}-\ell_{A_{2}, \psi_{1}^{\prime}}\right)\left(A_{1}\left(\psi_{2}\right)-A_{1}\left(\psi_{2}^{\prime}\right)\right)\right. \text {, } \\
& \left.-\mathscr{L}\left(\psi_{1}, A_{1}\left(\psi_{2}\right)-A_{1}\left(\psi_{2}^{\prime}\right)\right)-\ell_{A_{1}+A_{2}, \psi_{1}}^{*}\left(\ell_{F+A_{1}^{*}(w)+A_{2}^{*}(w)}^{*}\left(\psi_{2}^{\prime}\right)\right)\right), \\
& \tilde{A}_{1}\left(\tilde{B}_{2}^{*}\left(\left(\psi_{1}, \psi_{1}^{\prime}\right),\left(\psi_{2}, \psi_{2}^{\prime}\right)\right)\right) \\
& =\left(A_{1}\left(B_{2}^{*}\left(\psi_{1}, \psi_{2}\right)-B_{2}^{*}\left(\psi_{1}, \psi_{2}^{\prime}\right)-B_{2}^{*}\left(\psi_{1}^{\prime}, \psi_{2}\right)+B_{2}^{*}\left(\psi_{1}^{\prime}, \psi_{2}^{\prime}\right)\right)\right. \text {, } \\
& \left.\ell_{F+A_{1}^{*}(w)+A_{2}^{*}(w)}^{*}\left(B_{1}^{*}\left(\psi_{1}, \psi_{2}\right)+B_{2}^{*}\left(\psi_{1}, \psi_{2}^{\prime}\right)+B_{2}^{*}\left(\psi_{1}^{\prime}, \psi_{2}\right)-B_{2}^{*}\left(\psi_{1}^{\prime}, \psi_{2}^{\prime}\right)\right)\right) \text {, }
\end{aligned}
$$




$$
\begin{aligned}
& \tilde{A}_{2}\left(\tilde{B}_{1}^{*}\left(\left(\psi_{1}, \psi_{1}^{\prime}\right),\left(\psi_{2}, \psi_{2}^{\prime}\right)\right)\right) \\
& =\left(A_{2}\left(B_{1}^{*}\left(\psi_{1}, \psi_{2}\right)-B_{1}^{*}\left(\psi_{1}, \psi_{2}^{\prime}\right)-B_{1}^{*}\left(\psi_{1}^{\prime}, \psi_{2}\right)+B_{1}^{*}\left(\psi_{1}^{\prime}, \psi_{2}^{\prime}\right)\right),\right. \\
& \left.\quad-\ell_{F+A_{1}^{*}(w)+A_{2}^{*}(w)}^{*}\left(B_{1}^{*}\left(\psi_{1}, \psi_{2}\right)-B_{1}^{*}\left(\psi_{1}, \psi_{2}^{\prime}\right)-B_{1}^{*}\left(\psi_{1}^{\prime}, \psi_{2}\right)-B_{2}^{*}\left(\psi_{1}^{\prime}, \psi_{2}^{\prime}\right)\right)\right),
\end{aligned}
$$

were we introduced the notation $\mathscr{L}\left(\psi_{1}, \varphi_{2}\right)=\ell_{\ell_{F+A_{1}^{*}(w)+A_{2}^{*}(w)}, \psi_{1}}\left(\varphi_{2}\right)$. Let us set

$$
\left(\varphi_{3}, \psi_{3}^{\prime}\right)=\left[\tilde{A}_{1}, \tilde{A}_{2}\right]\left(\left(\psi_{1}, \psi_{1}^{\prime}\right),\left(\psi_{2}, \psi_{2}^{\prime}\right)\right) .
$$

We have

$$
\varphi_{3}=\llbracket\left[A_{1}, A_{2} \rrbracket\left(\psi_{1}, \psi_{2}\right)+\llbracket\left[A_{1}, A_{2}\right]\left(\psi_{1}^{\prime}, \psi_{2}^{\prime}\right)-\llbracket\left[A_{1}, A_{2} \rrbracket\left(\psi_{1}^{\prime}, \psi_{2}\right)-\left[\llbracket A_{1}, A_{2} \rrbracket\left(\psi_{1}, \psi_{2}^{\prime}\right)=0\right.\right.\right.
$$

because $\left[\left[A_{1}, A_{2}\right]\right]=0$.

As for the second component $\psi_{3}^{\prime}$, we first observe that the operator

$$
\mathscr{L}\left(\psi_{1}^{\prime}, \cdot\right): \varkappa \rightarrow \hat{\varkappa}
$$

is selfadjoint. Now, we take the adjoint of Eqs. 13) and compute them in $\psi_{2} \in \hat{P}$ using Eq. 8). We obtain

$$
\begin{aligned}
& \ell_{\ell_{F+A_{1}^{*}(w)}^{*}, \psi_{2}}^{*}\left(A_{1}\left(\psi_{1}\right)\right)+\ell_{A_{1}, \psi_{1}}^{*}\left(\ell_{F+A_{1}^{*}(w)}^{*}\left(\psi_{2}\right)\right) \\
& -\ell_{A_{1}, \psi_{2}}^{*}\left(\ell_{F+A_{1}^{*}(w)}^{*}\left(\psi_{1}\right)\right)-\ell_{\ell_{F+A_{1}^{*}(w)}^{*}, \psi_{1}}^{*}\left(A_{1}\left(\psi_{2}\right)\right)=\ell_{F+A_{1}^{*}(w)}^{*}\left(-B_{1}^{*}\left(\psi_{1}, \psi_{2}\right)\right) \\
& \ell_{\ell_{F+A_{1}^{*}(w)}^{*}, \psi_{2}}^{*}\left(A_{2}\left(\psi_{1}\right)\right)+\ell_{A_{2}, \psi_{1}}^{*}\left(\ell_{F+A_{1}^{*}(w)}^{*}\left(\psi_{2}\right)\right)-\ell_{A_{1}, \psi_{2}}^{*}\left(\ell_{A_{2}^{*}(w)}^{*}\left(\psi_{1}\right)\right)-\ell_{\ell_{A_{2}^{*}(w)}^{*}, \psi_{1}}^{*}\left(A_{1}\left(\psi_{2}\right)\right) \\
& -\ell_{A_{2}, \psi_{2}}^{*}\left(\ell_{F+A_{1}^{*}(w)}^{*}\left(\psi_{1}\right)\right)-\ell_{\ell_{F+A_{1}^{*}(w)}^{*}, \psi_{1}}^{*}\left(A_{2}\left(\psi_{2}\right)\right)+\ell_{\ell_{A_{2}^{*}(w)}^{*}, \psi_{2}}^{*}\left(A_{1}\left(\psi_{1}\right)\right)+\ell_{A_{1}, \psi_{1}}^{*}\left(\ell_{A_{2}^{*}(w)}^{*}\left(\psi_{2}\right)\right) \\
& =\ell_{F+A_{1}^{*}(w)}^{*}\left(-B_{2}^{*}\left(\psi_{1}, \psi_{2}\right)\right)+\ell_{A_{2}^{*}(w)}^{*}\left(-B_{1}\left(\psi_{1}, \psi_{2}\right)\right), \\
& \ell_{\ell_{A_{2}^{*}(w)}^{*}, \psi_{2}}^{*}\left(A_{2}\left(\psi_{1}\right)\right)+\ell_{A_{2}, \psi_{1}}^{*}\left(\ell_{A_{2}^{*}(w)}^{*}\left(\psi_{2}\right)\right)-\ell_{A_{2}, \psi_{2}}^{*}\left(\ell_{A_{2}^{*}(w)}^{*}\left(\psi_{1}\right)\right)-\ell_{\ell_{A_{2}^{*}(w)}^{*}, \psi_{1}}^{*}\left(A_{2}\left(\psi_{2}\right)\right) \\
& =\ell_{A_{2}^{*}(w)}^{*}\left(-B_{2}^{*}\left(\psi_{1}, \psi_{2}\right)\right) .
\end{aligned}
$$

Then, we sum the above three equations to get one single equation, that we compute two times in $\left(\psi_{1}, \psi_{2}^{\prime}\right)$ and $\left(\psi_{1}^{\prime}, \psi_{2}\right)$ respectively. By replacing the result into $\psi_{3}^{\prime}$ we obtain

$$
\begin{aligned}
\psi_{3}^{\prime}= & \mathscr{L}\left(\psi_{2}^{\prime}, A_{1}\left(\psi_{1}\right)+A_{2}\left(\psi_{1}^{\prime}\right)\right)+\mathscr{L}\left(\psi_{2}, A_{1}\left(\psi_{1}\right)+A_{2}\left(\psi_{1}^{\prime}\right)\right) \\
& -\mathscr{L}\left(\psi_{1}^{\prime}, A_{1}\left(\psi_{2}\right)+A_{2}\left(\psi_{2}^{\prime}\right)\right)-\mathscr{L}\left(\psi_{1}, A_{1}\left(\psi_{2}\right)+A_{2}\left(\psi_{2}^{\prime}\right)\right) .
\end{aligned}
$$

We define the operators

$$
\square_{1}, \square_{1}^{\prime}:(\hat{\varkappa} \times \varkappa) \times(\hat{P} \times \hat{P}) \rightarrow(\varkappa \times \hat{\varkappa}), \quad \square_{2}, \square_{2}^{\prime}:(\hat{P} \times \hat{P}) \times(\hat{\varkappa} \times \varkappa) \rightarrow(\varkappa \times \hat{\varkappa}),
$$

as follows:

$$
\begin{aligned}
& \square_{1}\left(\left(\varphi_{1}, \varphi_{1}^{\prime}\right),\left(\psi_{2}, \psi_{2}^{\prime}\right)\right)=\left(0,-\mathscr{L}\left(\psi_{2}^{\prime}, \varphi_{1}^{\prime}\right)\right), \quad \square_{2}\left(\left(\psi_{1}, \psi_{1}^{\prime}\right),\left(\varphi_{2}, \varphi_{2}^{\prime}\right)\right)=\left(0, \mathscr{L}\left(\psi_{1}^{\prime}, \varphi_{2}^{\prime}\right)\right), \\
& \square_{1}^{\prime}\left(\left(\varphi_{1}, \varphi_{1}^{\prime}\right),\left(\psi_{2}, \psi_{2}^{\prime}\right)\right)=\left(0, \mathscr{L}\left(\psi_{2}, \varphi_{1}^{\prime}\right)\right), \quad \square_{2}^{\prime}\left(\left(\psi_{1}, \psi_{1}^{\prime}\right),\left(\varphi_{2}, \varphi_{2}^{\prime}\right)\right)=\left(0,+\mathscr{L}\left(\psi_{1}, \varphi_{2}^{\prime}\right)\right) .
\end{aligned}
$$

We have

$$
\begin{aligned}
\left(0, \psi_{3}^{\prime}\right) & =\square_{1}\left(\ell_{\tilde{\mathscr{E}}}^{*}\left(\psi_{1}, \psi_{1}^{\prime}\right),\left(\psi_{2}, \psi_{2}^{\prime}\right)\right)+\square_{2}\left(\left(\psi_{1}, \psi_{1}^{\prime}\right), \ell_{\tilde{\mathscr{E}}}^{*}\left(\psi_{2}, \psi_{2}^{\prime}\right)\right) \\
& +\square_{1}^{\prime}\left(\ell_{\tilde{\mathscr{E}}}^{*}\left(\psi_{1}, \psi_{1}^{\prime}\right),\left(\psi_{2}, \psi_{2}^{\prime}\right)\right)+\square_{2}^{\prime}\left(\left(\psi_{1}, \psi_{1}^{\prime}\right), \ell_{\tilde{\mathscr{E}}}^{*}\left(\psi_{2}, \psi_{2}^{\prime}\right)\right),
\end{aligned}
$$

hence the three-vector $\left[\left[\tilde{A}_{1}, \tilde{A}_{2}\right]\right]$ is zero up to trivial terms. 
Theorem 2 Let $\omega_{1}, \omega_{2}, \ldots$ is a Magri hierarchy for $\mathscr{E}$. Suppose that there exist extensions of $\omega_{i}, A_{1}$, and $A_{2}$ onto $J^{\infty}$ such that on $J^{\infty}$ we have

$$
\begin{aligned}
\bar{d} \omega_{i} & =\left\langle\psi_{i}, F\right\rangle, \\
A_{1}\left(\psi_{i}\right) & =A_{2}\left(\psi_{i+1}\right),
\end{aligned}
$$

here $\psi_{i}$ is an extension of the generating function of $\omega_{i}$. Then $\left(\psi_{i},-\psi_{i+1}\right), i=1,2, \ldots$ is a Magri hierarchy for the Kupershmidt deformation $\tilde{\mathscr{E}}$.

Proof On $\tilde{J}^{\infty}$ we have

$$
\begin{aligned}
\bar{d} \omega_{i}=\left\langle\psi_{i}, F\right\rangle=\left\langle\psi_{i}, F\right. & \left.+A_{1}^{*}(w)\right\rangle-\left\langle\psi_{i}, A_{1}^{*}(w)\right\rangle \\
& =\left\langle\psi_{i}, F+A_{1}^{*}(w)\right\rangle-\left\langle A_{1}\left(\psi_{i}\right), w\right\rangle+\bar{d} \chi_{1} \\
= & \left\langle\psi_{i}, F+A_{1}^{*}(w)\right\rangle- \\
& \left\langle A_{2}\left(\psi_{i+1}\right), w\right\rangle+\bar{d} \chi_{1} \\
& =\left\langle\psi_{i}, F+A_{1}^{*}(w)\right\rangle-\left\langle\psi_{i+1}, A_{2}^{*}(w)\right\rangle+\bar{d} \chi_{2} .
\end{aligned}
$$

Thus, the form $\omega_{i}-\chi_{2}$ is a conservation law with the generating function $\left(\psi_{i},-\psi_{i+1}\right)$. The condition $\tilde{A}_{1}\left(\psi_{i},-\psi_{i+1}\right)=\tilde{A}_{2}\left(\psi_{i+1},-\psi_{i+2}\right)$ can be easily checked by direct computation.

Acknowledgements We wish to thank Sergey Igonin for reading the manuscript and useful comments.

\section{References}

1. Bocharov, A.V., Chetverikov, V.N., Duzhin, S.V., Khor'kova, N.G., Krasil'shchik, I.S., Samokhin, A.V., Torkhov, Y.N., Verbovetsky, A.M., Vinogradov, A.M.: Symmetries and Conservation Laws for Differential Equations of Mathematical Physics. Monograph. Amer. Math. Soc. (1999)

2. Igonin, S., Kersten, P., Krasilshchik, I., Verbovetsky, A., Vitolo, R.: Variational brackets in the geometry of PDEs (2009). To appear

3. Karasu-Kalkanlı, A., Karasu, A., Sakovich, A., Sakovich, S., Turhan, R.: A new integrable generalization of the Korteweg-de Vries equation. J. Math. Phys. 49, 073516 (2008), arXiv:0708.3247

4. Kersten, P., Krasil'shchik, I., Verbovetsky, A., Vitolo, R.: Hamiltonian structures for general PDEs. In: B. Kruglikov, V.V. Lychagin, E. Straume (eds.) Differential equations: Geometry, Symmetries and Integrability. The Abel Symposium 2008. Springer-Verlag (2009)

5. Krasil'shchik, J., Verbovetsky, A.M.: Homological Methods in Equations of Mathematical Physics. Advanced Texts in Mathematics. Open Education \& Sciences, Opava (1998), arXiv:math/9808130

6. Kupershmidt, B.A.: KdV6: An integrable system. Phys. Lett. A 372, 2634-2639 (2008), arXiv:0709.3848

7. Vinogradov, A.M.: Cohomological Analysis of Partial Differential Equations and Secondary Calculus. Amer. Math. Soc. (2001) 